\title{
Interaction of Nanoparticles with Ideal Liquid-Liquid Interfaces
}

\author{
David L. Cheung* \\ Department of Chemistry and Centre for Scientific Computing, University of Warwick, Coventry, CV4 7AL, United Kingdom
}

Stefan A. F. Bon ${ }^{\dagger}$

Department of Chemistry, University of Warwick, Coventry, CV4 7AL, United Kingdom

(Received 15 August 2008; published 13 February 2009)

\begin{abstract}
Using molecular simulations the interaction between a noncharged nanoparticle and an ideal liquidliquid interface is studied. The free energy profile as function of nanoparticle-interface separation is determined using Wang-Landau sampling. Comparison between the simulation results and macroscopic theories shows that the latter give a poor description of the free energy profile. In particular, they underestimate both the range of interaction between the particle and the interface and its strength, with the discrepancy lessening as the particle radius increases. On increasing the solvent chemical potential the interaction strength increases and the interaction range decreases due to the increase in interfacial tension and consequent decrease in interfacial width.
\end{abstract}

DOI: 10.1103/PhysRevLett.102.066103

PACS numbers: 68.05.Cf, 05.20.Jj, 68.03.Cd

Nanoparticles have attracted a great deal of interest as building blocks for materials with a range of applications [1]. In order to realize many of these, nanoparticles often have to be arranged into specific structures. Because of the impracticality of direct manipulation of nm-sized particles, there is much investigation of their self-assembly into larger structures, with a range of methods studied [2]. One particularly successful method is adhesion on to fluid interfaces [3] and using this approach a variety of supracolloidal structures have been reported, such as permeable hollow capsules made from partially fused polymer latexes (colloidosomes) [4], colloid-based foams [5] and gels [6] and rigid cellular materials [7,8], crosslinked nanoparticle membranes [9], and nanocomposite armored polymer latexes [10]. The assembly of small objects at soft interfaces extends into many biological contexts, for example, the fabrication of capsules via directed self-assembly and cross-linking of virus bionanoparticles [11] and the aggregation of crystalline arrays of proteins in bacterial $S$ layers [12].

The underlying phenomenon of particle adhesion to liquid interfaces was first described over a century ago $[13,14]$. In the absence of charges or applied external fields the binding of particles to liquid interfaces is explained in terms of the wettability of particles and changes in surface areas $[15,16]$ and the free energy as a function of particleinterface separation may then be found on purely geometric grounds. An expression for a spherical particle of radius $R_{c}$, extended to account for line tension, was given by Aveyard and Clint (AC) [17]

$$
\begin{aligned}
F(\bar{z})= & -\pi R_{c}^{2} \gamma_{12}\left(1-\bar{z}^{2}\right)+2 \pi R_{c}^{2}\left(\gamma_{1 P}-\gamma_{2 P}\right)(1-\bar{z}) \\
& +2 \pi R_{c} \tau \sqrt{1-\bar{z}^{2}}, \quad|\bar{z}| \leq 1
\end{aligned}
$$

where $\bar{z}=z / R_{c} \cdot \gamma_{i P}$ is the interfacial tension between component $i$ and the particle, $\gamma_{12}$ is the interfacial tension between components 1 and 2, and $\tau$ is the line tension. Such a description has been used to successfully model the binding of $\mu \mathrm{m}$-sized particles onto fluid interfaces. Particular attention has been paid to the effect of the line tension [the final term in Eq. (1)], which becomes increasingly important for decreasing $R_{c}$ [17].

As these models are formulated in terms of macroscopic quantities the question arises about their validity when scaled down to nm-sized objects. Because of their small size the interaction between the nanoparticles and liquid interfaces is expected to be somewhat more complex [18] and the binding of nanoparticles to liquid interfaces is also much weaker (typically $1-100 k_{B} T$ ). Competition between these adhesion forces and Brownian motion gives rise to reversibility and size selectivity of the self-assembly process, which has been demonstrated in experimental studies of mixtures of Laponite clay discs [10] and CdSe quantum dots $[9,19]$. Previous simulation studies have indicated that, with the introduction of size dependent surface and line tensions, Eq. (1) may describe the binding strength of nanoparticles to fluid interfaces [20-22]. However, these did not probe the interaction potential between the nanoparticle and interface, which due to capillary waves and other microscopic effects may be different to that predicted by macroscopic theories [23] and is important in understanding both the self-assembly and dynamics of nanoparticles near interfaces.

In this Letter we determine the interaction between a model nanoparticle and a liquid-liquid interface. The solvent is modeled as a two-component WidomRowlinson (WR) fluid [24], with the interaction between solvent molecules of species $\alpha$ and $\beta$ given by $u(r)=$ $\left(1-\delta_{\alpha \beta}\right) u_{\mathrm{hs}}(r)$ with $\alpha, \beta=1,2$, where $u_{\mathrm{hs}}(r)$ is the potential for hard spheres of diameter $\sigma$ and $\delta_{\alpha \beta}$ is the Kronecker delta function. Above a critical density $\left(\rho_{c} \sigma^{3} \approx 0.75\right.$ [25]) this demixes into 1 and 2 rich regions. 
It should be noted that this model neglects electrostatic and attractive van der Waals interactions present in real systems. However, due to its simplicity it is an attractive model for studying interfaces and a number of recent studies [25-27] have investigated its phase behavior and interfacial properties. The nanoparticle is modeled as a hard sphere of radius $R_{c}=1.5 \sigma$ to $R_{c}=3 \sigma$; the fluidnanoparticle interaction is identical for both components.

The system is simulated using grand-canonical (constant- $\mu V T$ ) Monte Carlo simulations. For this model the temperature is not a significant variable (and is set to $k_{B} T=1 / \beta=1$ ), and the phase behavior is controlled solely by the chemical potential (or equivalently by changes in the solvent density $\rho$ ). The surface and line tensions and the interfacial width are also functions of $\mu$. Simulations were performed at $\beta \mu=0.15,0.20,0.25$, 0.30 , and 0.35 (at $\rho_{c} \beta \mu \approx 0.04$ ). In order to localize the interface near the cell center, the fluid is confined between two walls in the $z$ direction (periodic boundaries are applied in the $x$ and $y$ directions). Systems of lateral dimension $L_{x}=L_{y}=10 \sigma, 15 \sigma$, and $20 \sigma$ were studied and the separation between the walls was $L_{z}=30 \sigma$, except for the $R_{c}=3 \sigma$ nanoparticle, for which $L_{z}=40 \sigma$ was used.

The free energy profile for the nanoparticle is found using $F\left(z_{c}\right)=-k_{B} T \log \mathcal{P}\left(z_{c}\right)$, where $\mathcal{P}\left(z_{c}\right)$ is the probability distribution and $z_{c}$ is the nanoparticle-interface separation. The position of the interface, $z_{0}$, is found from maximizing the function $f\left(z_{0}\right)=\sum_{i} q_{i} \cos \left\{\left(\frac{2 \pi}{L}\right)\left(z_{i}+z_{0}\right)\right\}$ [26], where $q_{i}=1$ for 1 and $q_{i}=-1$ for 2 . $z_{0}$ and the nanoparticle-interface separation are updated after every MC move. It should be noted that this gives the average interface position across the simulation cell. Recently a number of papers have attempted to formulate definitions of the intrinsic surface (in the absence of capillary waves) [28]. In order to sample $\mathcal{P}(z)$ effectively the $z$ separation is divided into a set of overlapping windows of width $2 \sigma$. Within each window a weighting function $w(z)$, determined iteratively using the Wang-Landau algorithm [29], is applied to give a flat distribution. $w(z)$ was determined over up to $5 \times 10^{6} \mathrm{MC}$ sweeps [30] and a (weighted) probability distribution $\mathcal{P}_{\text {sim }}(z)$ is found from a production run of $10^{6} \mathrm{MC}$ sweeps. The true probability distribution for each window may be recovered from $P(z)=P_{\text {sim }}(z) \times$ $\exp [\beta w(z)]$, and the full distribution is found using the weighted-histogram analysis method [31].

For comparison with macroscopic expression the interfacial tension $\gamma_{12}$ was determined from simulations without a nanoparticle from $\gamma_{12}=\int d z P_{N}(z)-P_{T}(z)$ [32], where $P_{N}(z)=P_{z z}(z)$ and $P_{T}(z)=P_{x x}(z)=P_{y y}(z)$. As the nanoparticle-fluid interaction is the same for both components, $\gamma_{1 P}=\gamma_{2 P}$ and Eq. (1) reduces to

$$
\begin{array}{rlr}
F(\bar{z})= & -\pi R_{c}^{2}\left(1-\bar{z}^{2}\right) \gamma_{12} & \\
& +2 \pi R_{c} \sqrt{\left(1-\bar{z}^{2}\right)} \tau, & |\bar{z}| \leq 1, \\
F(\bar{z})= & -\pi R_{c}^{2}\left(1-\bar{z}^{2}\right) \gamma_{12}, & |\bar{z}| \leq 1,
\end{array}
$$

TABLE I. Densities, surface tensions, and interface widths for WR mixture $\left(L_{z}=30 \sigma\right)$. Uncertainites in the final digits are shown in parenthesis. For $L_{z}=40 \sigma \quad\left(L_{x}=15 \sigma\right) \rho \sigma^{3}=$ 1.066(6), $\beta \sigma^{2} \gamma_{12}=0.172(1)$ and $w=2.95 \sigma$ at $\beta \mu=0.15$.

\begin{tabular}{ccccccc}
\hline \hline & \multicolumn{3}{c}{$L_{x}=10 \sigma$} & \multicolumn{3}{c}{$L_{x}=15 \sigma$} \\
$\beta \mu$ & $\rho \sigma^{3}$ & $\beta \sigma^{2} \gamma_{12}$ & $w / \sigma$ & $\rho \sigma^{3}$ & $\beta \sigma^{2} \gamma_{12}$ & $w / \sigma$ \\
\hline 0.15 & $1.056(4)$ & $0.173(6)$ & 2.60 & $1.056(6)$ & $0.167(3)$ & 3.00 \\
0.20 & $1.128(7)$ & $0.226(6)$ & 2.28 & $1.128(8)$ & $0.220(2)$ & 2.61 \\
0.25 & $1.200(6)$ & $0.276(8)$ & 2.05 & $1.200(7)$ & $0.270(2)$ & 2.29 \\
0.30 & $1.274(5)$ & $0.335(6)$ & 1.80 & $1.274(5)$ & $0.328(1)$ & 2.06 \\
0.35 & $1.350(5)$ & $0.394(8)$ & 1.65 & $1.350(5)$ & $0.391(2)$ & 1.87 \\
\hline \hline
\end{tabular}

where the second form, which neglects line tension, is the expression reported by Pieranski [16].

The effect of capillary waves gives the interface a finite width $w$. This may be found from the decay of the solvent density profiles (averaged over the simulations), with $w=$ $-[x(Z)-x(-Z)]\left\{\frac{d x(z)}{d z}\right\}_{z=z_{0}}^{-1} \quad[26]$, where $x(z)=\rho_{1}(z) /$ $\left[\rho_{1}(z)+\rho_{2}(z)\right]$ and $x( \pm Z)$ are the average bulk compositions away from the interface $(Z=10 \sigma)$. The calculated values of $\rho, \gamma_{12}$, and $w$ are listed in Table I and are in good agreement with previously calculated values [25,26].

Shown in Fig. 1 are representative trajectories of the nanoparticle for unweighted simulations. For $R_{c}=1.5 \sigma$ the nanoparticle rapidly detaches from the interface and gets trapped near a wall. This occurs at both $\beta \mu=0.15$ and $\beta \mu=0.30$, although in the latter case the detachment from the interface takes longer (it occurs after approximately $50000 \mathrm{MC}$ steps, whereas at $\beta \mu=0.15$ detachment occurs within $10000 \mathrm{MC}$ steps), and the nanoparticle briefly rejoins the interface. By contrast the $R_{c}=2.5 \sigma$ nanoparticle remains in contact with the interface for at least 10 times more MC sweeps, consistent with experimental studies $[9,19]$.

The nanoparticle-interface potentials for $\beta \mu=0.15$ for a range of nanoparticle radii are shown in Fig. 2(a). The potentials have minima at $z_{c}=0$, while at larger separa-

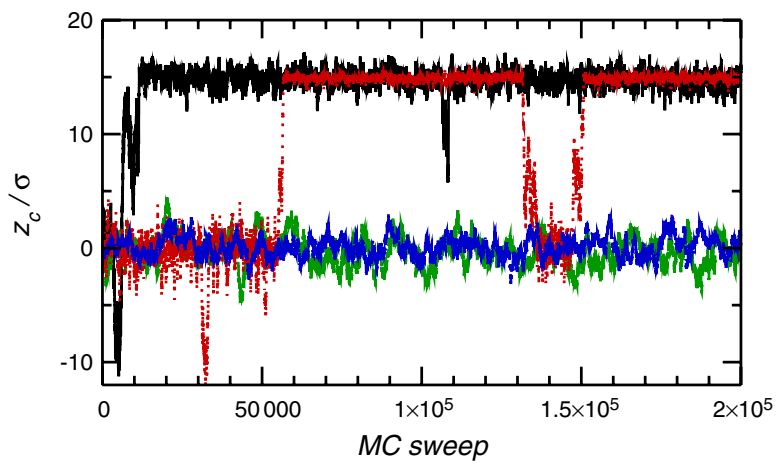

FIG. 1 (color online). Nanoparticle-interface separation for unbiased simulations for $R_{c}=1.5 \sigma$ nanoparticle at $\beta \mu=$ 0.15 (solid line, black) and $\beta \mu=0.30$ (dotted line, red) and $R_{c}=2.5 \sigma$ nanoparticle at $\beta \mu=0.15$ (dashed line, green) and $\beta \mu=0.30$ (dot-dashed line, blue). 

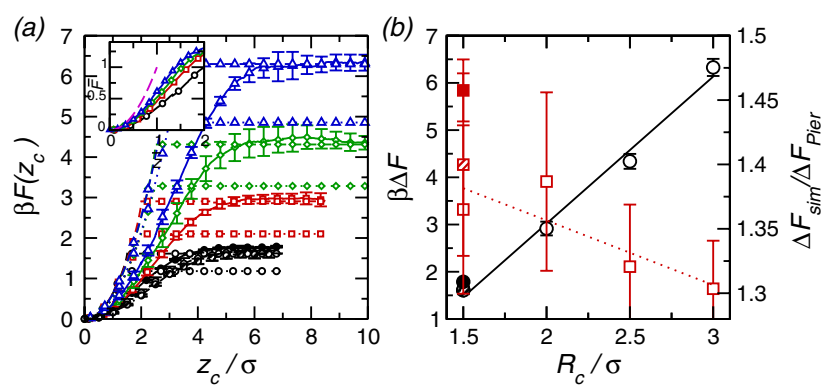

FIG. 2 (color online). (a) Free energy profiles $\left(z_{c} \geq 0\right)$ for $\beta \mu=0.15$ and nanoparticle radius $R_{c}=1.5 \sigma$ (circles, black), $R_{c}=2.0 \sigma$ (squares, red), $R_{c}=2.5 \sigma$ (diamonds, green), and $R_{c}=3.0 \sigma$ (triangles, blue). In all cases, solid line denotes simulation profile and dotted line profile from Eq. (2b). The inset shows reduced free energy $\bar{F}=\beta F(z) / \pi R_{c}^{2} \gamma_{12}$ (dotted line shows $\bar{F}=\bar{z}^{2}$ ). (b) Binding free energy (circles, black) and $\Delta F / \Delta F_{\text {Pier }}$ (squares, red) against $R_{c}$ for $\beta \mu=0.15$. For the $R_{c}=1.5 \sigma$ nanoparticle, the filled, open, and striped symbols denote $L_{x}=10 \sigma, 15 \sigma$, and $20 \sigma$.

tions there is a point of inflection in the potential, which then decays to a constant value. For the smallest particle the binding energy [Fig. 2(b)] is comparable to the thermal energy $\left[\Delta \beta F\left(R_{c}=1.5 \sigma\right) \approx 1.8\right]$, which is consistent with the rapid detachment of the particle from the interface in the unbiased simulations (Fig. 1). The binding energies for all $R_{c}$ studied are within the range expected $\left(1-10 k_{B} T\right)$ for nm-sized particles [19].

For the particle sizes studied the free energy barrier increases quadratically with $R_{c}$, in agreement with the Pieranski expression $\left(\Delta F_{\text {Pier }}=\pi R_{c}^{2} \gamma_{12}\right)$ [Eq. (2b)], but with an additional contribution that is seemingly linear in $R_{c}$. On increasing $R_{c}$ the ratio between the simulation and Pieranski binding energies decreases [Fig. 2(b)], as may be expected. The linear $R_{c}$ dependence and the difference between the simulation and predicted binding free energies may be explained through a nonzero line tension. $\tau$ may be determined by fitting to the simulation $\Delta F$ [20-22]. For all $R_{c} \tau$ is negative and its magnitude increases with $R_{c}$ (Table. II). Previous simulations [22] also found a negative $\tau$ for small $R_{c}$, with a change in sign on increasing nanoparticle radius.

From Fig. 2(a) it may be seen that the range of interaction is somewhat larger than predicted by Eqs. (2a) and (2b). This underestimation of the interaction range follows

TABLE II. Fitted values of line tension. Errors in final digits given in paraenthesis.

\begin{tabular}{ccccc}
\hline \hline \multicolumn{5}{c}{$\beta \sigma \tau$} \\
$\beta \mu$ & $R_{c}=1.5 \sigma$ & $R_{c}=2 \sigma$ & $R_{c}=2.5 \sigma$ & $R_{c}=3 \sigma$ \\
\hline 0.15 & $-0.06(1)$ & $-0.06(2)$ & $-0.10(2)$ & $-0.09(2)$ \\
0.20 & $-0.06(1)$ & $-0.07(1)$ & $-0.08(2)$ & $\ldots$ \\
0.25 & $-0.06(1)$ & $-0.07(1)$ & $-0.08(1)$ & $\ldots$ \\
0.30 & $-0.06(1)$ & $-0.07(1)$ & $-0.10(1)$ & $\ldots$ \\
0.35 & $-0.05(1)$ & $-0.05(1)$ & $-0.07(1)$ & $\ldots$ \\
\hline \hline
\end{tabular}

from their neglect of capillary waves (i.e., assuming a flat interface). The effect of these is shown in the simulation snapshots in Fig. 3. In the absence of the nanoparticle [Fig. 3(a)] capillary waves broaden the interface, leading to a diffuse density profile across the interface (although on the level of individual fluid molecules the interface is still sharp). For the smallest particle the size of these fluctuations are comparable to the particle size [Fig. 3(b)], as $w \approx 2 R_{c}$, while the largest particle spans the interface [Fig. 3(c)]. When $z_{c}>R_{c}$ interface fluctuations may lead to bridging between the particle and interface [Fig. 3(d)], leading to a longer-ranged interaction, showing that the effect of capillary waves is vital to the accurate description of nm-sized particles at interfaces. On increasing $R_{c}$ the effect of these capillary waves becomes smaller. Shown in the inset of Fig. 2(a) is the reduced free energy $\bar{F}(z)=$ $\beta F(z) / \pi R_{c}^{2} \gamma_{12}$; on increasing $R_{c}$ this narrows and tends towards the continuum description.

Both Eqs. (2a) and (2b) predict that around $z_{c}=0$ the nanoparticle-interface interaction varies more rapidly with separation than simulation. The variation in the free energy profile may be measured by the spring constant $k=$ $d^{2} F /\left.d z^{2}\right|_{z=0}$. This increases approximately linearly with $R_{c}$ with $k \beta \sigma^{2}=0.40,0.53,0.67$, and 0.84 for $R_{c}=1.5 \sigma$, $2.0 \sigma, 2.5 \sigma$, and $3.0 \sigma$, respectively. From Eq. (2b), $k=$ $2 \pi R_{c}^{2} \gamma_{12}$, which is between 1.5 and 3 times larger than that from simulation (with the difference decreasing with increasing $R_{c}$ ). $k$ from the $\mathrm{AC}$ expression [Eq. (2a)] are larger than those from Eq. (2b), due to the negative line tensions. Recently it has been shown that the inclusion of capillary waves leads to a reduction in the steepness of the potential well [23].

In order to test the system size dependence of the free energy profile has been calculated for the $R_{c}=1.5 \sigma$ nanoparticle for cells with $L_{x}=10 \sigma, 15 \sigma$, and $20 \sigma$. On increasing the lateral dimension the free energy profile broadens and $\Delta F$ decreases [Fig. 2(a)]. These are both consistent with the decrease in $\gamma_{12}$ with increasing $L_{x}$. The ratio $\Delta F_{\text {sim }} / \Delta F_{\text {Pier }}$ shows no systematic variation with $L_{x}$ (although results for $L_{x}=15 \sigma$ and $20 \sigma$ are equal

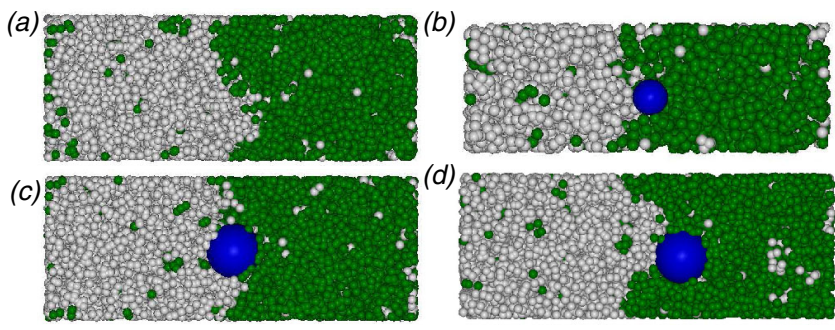

FIG. 3 (color online). Simulation snapshots at solvent chemical potential $(\beta \mu=0.15)$. (a) Nanoparticle free mixture, (b) $R_{c}=1.5 \sigma$ nanoparticle at separation $z_{c}=0.95 \sigma$, (c) $R_{c}=3 \sigma$ nanoparticle at separation $z_{c}=-0.13 \sigma$, (d) $R_{c}=3 \sigma$ nanoparticle at separation $z_{c}=3.7 \sigma$. Snapshots produced using the QMGA package [33]. 

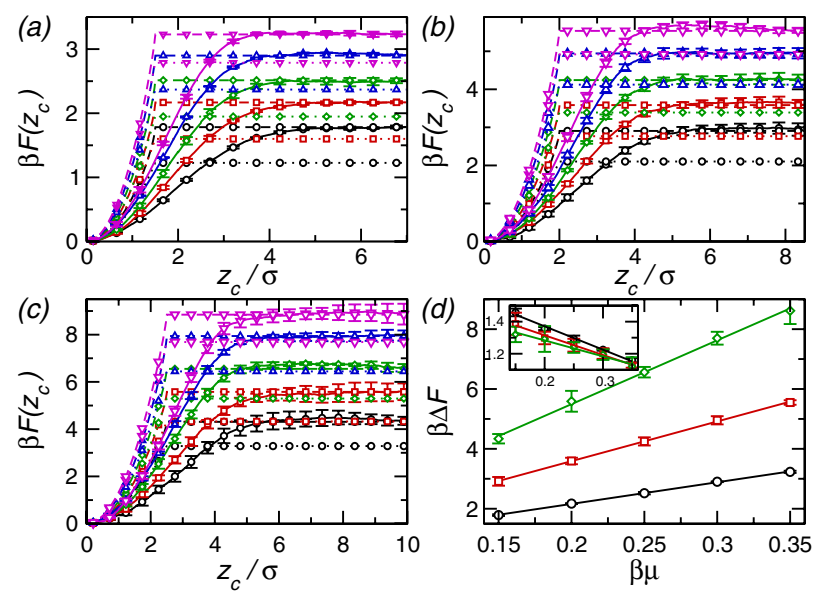

FIG. 4 (color online). Free energy profiles for (a) $R_{c}=1.5 \sigma$, (b) $R_{c}=2.0 \sigma$, and (c) $R_{c}=2.5 \sigma$ nanoparticles. In all cases $\beta \mu=0.15$ denoted by (circles, black), $\beta \mu=0.20$ (squares, red), $\beta \mu=0.25$ (diamonds, green), $\beta \mu=0.30$ (triangles, blue), and $\beta \mu=0.35$ (inverted triangles, magenta). Solid line denotes simulation profiles and dotted line denotes Eq. (2b). (d) $\beta \Delta F$ (inset $\Delta F / \Delta F_{\text {Pier }}$ ) against $\beta \mu$ for $R_{c}=1.5 \sigma$ (circles), $2.0 \sigma$ (squares), and $2.5 \sigma$ (diamonds) nanoparticles.

within the statistical error), which suggests that the present results are robust against changes in $L_{x}$.

The potential as a function of $\mu$ for varying $R_{c}$ is shown in Fig. 4. The interaction strength [Fig. 4(d)] increases with $\mu$, which arises due to the linear dependence of $\gamma_{12}$ on $\mu$ (Table I). The fitted $\tau$ remain constant (within statistical errors) so the ratio between the simulation and Pieranski binding energies decreases with $\mu$ [shown in the inset in Fig. 4(d)]. On increasing $\mu$ the interaction range decreases, due to the decrease in capillary wave amplitude with $\gamma_{12}$ (reflected in the decrease in $w$ ).

In conclusion, the interaction between a fluid interface and a nanoparticle has been calculated using MC simulations. This interaction is attractive with a binding strength in the range $1-10 k_{B} T$, consistent with experimental observations [9]. The binding energy was found to increase quadratically with $R_{c}$, but with an additional linear dependence on $R_{c}$, while the range of interaction is significantly larger than $R_{c}$. The macroscopic approximations substantially underestimate the interaction range, likely due to their neglect of capillary waves. The binding energy increases approximately linearly with $\mu$ as a consequence of the increase in the interfacial tension, while the range decreases. On increasing $\gamma_{12}$ (via increasing $\mu$ ) the simulation binding energy approaches the prediction of Pieranski approximation, indicating that this is approximately accurate for high interfacial tensions.

The authors wish to thank Professor Michael Allen and Dr. Alessandro Troisi for advice and helpful comments during this work. Computational resources were provided by the Centre for Scientific Computing, University of Warwick and this work was funded by UK EPSRC. *david.cheung@warwick.ac.uk

†s.bon@warwick.ac.uk

[1] G. A. Ozin and A. Arsenault, Nanochemistry: A Chemical Approach to Nanomaterials (RSC, Cambridge, U.K., 2005).

[2] S. Kinge, M. Crego-Calama, and D. N. Reinhoudt, Chem. Phys. Chem. 9, 20 (2008).

[3] A. Böker et al., Soft Matter 3, 1231 (2007).

[4] A. D. Dinsmore et al., Science 298, 1006 (2002).

[5] B.P. Binks and K. Murakami, Nature Mater. 5, 865 (2006).

[6] P. S. Clegg, J. Phys. Condens. Matter 20, 113101 (2008).

[7] A. Menner et al., Chem. Commun. (Cambridge) 41, 4274 (2007).

[8] P. J. Colver and S. A. F. Bon, Chem. Mater. 19, 1537 (2007).

[9] Y. Lin et al., Science 299, 226 (2003).

[10] S. A. F. Bon and P. J. Colver, Langmuir 23, 8316 (2007).

[11] J. T. Russell et al., Angew. Chem. 44, 2420 (2005).

[12] U. B. Sleytr et al., FEBS J. 274, 323 (2007).

[13] W. Ramsden, Proc. R. Soc. A 72, 156 (1903).

[14] S. U. Pickering, J. Chem. Soc. Trans. 91, 2001 (1907).

[15] P. Finkle, H.D. Draper, and J.H. Hildebrand, J. Am. Chem. Soc. 45, 2780 (1923).

[16] P. Pieranski, Phys. Rev. Lett. 45, 569 (1980).

[17] R. Aveyard and J. H. Clint, J. Chem. Soc., Faraday Trans. 92, 85 (1996).

[18] F. Bresme and M. Oettel, J. Phys. Condens. Matter 19, 413101 (2007).

[19] Y. Lin et al., Langmuir 21, 191 (2005).

[20] F. Bresme and N. Quirke, Phys. Rev. Lett. 80, 3791 (1998).

[21] F. Bresme and N. Quirke, J. Chem. Phys. 110, 3536 (1999).

[22] F. Bresme and N. Quirke, Phys. Chem. Chem. Phys. 1, 2149 (1999).

[23] H. Lehle and M. Oettel, J. Phys. Condens. Matter 20, 404224 (2008).

[24] B. Widom and J. S. Rowlinson, J. Chem. Phys. 52, 1670 (1970).

[25] E. de Miguel, N. G. Almarza, and G. Jackson, J. Chem. Phys. 127, 034707 (2007).

[26] A. Malijevský and S. Sokołowski, J. Chem. Phys. 126, 106101 (2007).

[27] Y. Djikaev, J. Chem. Phys. 128, 014712 (2008).

[28] E. Chaćon and P. Tarazona, J. Phys. Condens. Matter 17, S3493 (2005).

[29] F. G. Wang and D. P. Landau, Phys. Rev. Lett. 86, 2050 (2001).

[30] One sweep consists of 3000-10000 translations, split equally between the solvent molecules and nanoparticle, 500 particle identity swaps, and 500 insertions and deletions.

[31] A. M. Ferrenberg and R. H. Swendsen, Phys. Rev. Lett. 63, 1195 (1989).

[32] J. H. Irving and J. G. Kirkwood, J. Chem. Phys. 18, 817 (1950).

[33] A. T. Gabriel, T. Meyer, and G. Germano, J. Chem. Theory Comput. 4, 468 (2008). 